\title{
Special issue on "Innovative approaches of softcomputing to networking systems and applications"
}

\author{
Fatos Xhafa ${ }^{1}$ - Leonard Barolli ${ }^{2}$
}

Published online: 29 August 2017

(C) Springer-Verlag GmbH Germany 2017

Networking systems of today pose many challenging research questions, which are requiring the use of techniques from other domains to satisfactorily solve them. Indeed, the resolution of such problems has become among most rich and innovative interdisciplinary research fields. Without no doubts, softcomputing techniques, such as evolutionary algorithms and genetic programming, neural science and neural net systems and fuzzy systems are of utmost importance to solve problems from networking systems.

This special issue comprises four high-quality contributions proposing innovative softcomputing techniques for solving relevant problems from networking systems to make them more efficient, optimal, intelligent, reliable, secure, and trusted. The integration of such softcomputing solutions into networking system is as well addressed. The papers of the special issue are arranged as follows.

The first paper "The Problem Aware Local Search Algorithm: An Efficient Technique for Permutation-based Problems" by Minetti et al. examines whether the Problem Aware Local Search (PALS) is useful in other application domains. The authors carry out a comprehensive analysis by using a wide benchmark of well-known problems with different kinds of variation operators and fitness functions, such as the quadratic assignment problem, the flow shop scheduling problem, and the multiple knapsack problem. It is shown that, in general, PALS can efficiently achieve high-quality solutions for these problems.

$凶$ Fatos Xhafa

fatos@1si.upc.edu

Leonard Barolli

barolli@ fit.ac.jp

1 Universitat Politècnica de Catalunya, Barcelona, Spain

2 Fukuoka Institute of Technology, Fukuoka, Japan
Yu et al. in the second paper "Real-time Processing of $k$ NN Queries over Moving Objects" investigate the task of processing k-nearest neighbor $(\mathrm{k}-\mathrm{NN})$ queries over moving objects. The aim is to overcome limitations of many existing approaches of search algorithms based on different index structures, whose maintenance cost is high and performance is not predictable. The authors present a dynamic Strip-Rectangle Index (SRI), which can reach a good balance between the maintenance cost and the performance of supporting k-NN queries over moving objects. An experimental study and empirical results are presented to evaluate the performance of the proposal and to support the claims.

In the third paper "Low-Power Sensing Model Considering Context Transition for Location-Based Services" Park et al. study the issue of the provision of sustainable context awareness, namely the transition between contexts aiming to overcome limitations of handling each context individually. The authors consider the representative case of indoor/outdoor contexts. The authors propose a low-power sensing model capable of considering context transition for a location-based service. By applying their method to context-aware services for indoor/outdoor contexts, the power efficiency is improved by $60 \%$ in the unstable state.

Finally, Sohn et al. in the last paper "Crowdsourced Healthcare Knowledge Creation using Patients' Health Experience-Ontologies" present a framework for creation and integration of crowdsourced healthcare knowledge using experience ontologies. The purpose is to provide patients' healthcare information which contains similar healthcare experiences including conditions and symptoms and integrates the features and relations in the particular patients' data according users' queries. Two experiments were conducted to evaluate the effectiveness of the proposed framework. The conducted experiments proved the efficiency of framework 
by the reduction of search volumes and the improvement of accuracy of query results.

Acknowledgements The guest editors would like to thank Prof. Vincenzo Loia (Editor-in-Chief of SoftComputing) for the opportunity to edit this special issue. We would like to thank all authors, reviewers and editorial members for their invaluable contribution. Finally, we appreciate the Journal Manager for the attention and support during the special issue editing process.

\section{List of papers}

1. The Problem Aware Local Search Algorithm: An Efficient Technique for Permutation-based Problems Gabriela F. Minetti, Gabriel Luque, Enrique Alba
2. Real-time Processing of k-NN Queries over Moving Objects

Ziqiang Yu, Yuehui Chen, Kun Ma

3. Low-Power Sensing Model Considering Context Transition for Location-Based Services Jae-Hyeon Jae-Hyeon Park, Deok-ki Kim, Dusan Baek, Jung-Won Lee

4. Crowdsourced Healthcare Knowledge Creation using Patients' Health Experience-Ontologies

Mye Sohn, Sunghwan Jeong, Jongmo Kim, Hyun Jung Lee 